\title{
A NEW PROOF OF CERTAIN RELATIONS OF MORSE IN THE CALCULUS OF VARIATIONS IN THE LARGE*
}

BY D. E. RICHMOND

1. Introduction. In a recent paper, $\dagger$ M. Morse has developed a theory of calculus of variations "in the large," in which he has made use of the results of his paper $\ddagger$ on critical points of functions of $n$ variables. He establishes a parallelism between types of critical points and types of extremal arcs in which the type of an extremal arc whose end points are not conjugate is completely determined by the number of mutual conjugate points within the arc. As a first application of the critical point theory, he obtains a set of necessary relations between the numbers of extremal arcs of different type, which join two given points. He also discusses periodic extremals as to type, and obtains number relations from the critical point theory.

Morse's relations are obtained for the parametric problem under certain general boundary conditions and under the hypothesis of the existence of a field of extremals covering the region of operation.

The present paper treats only the number relations between extremal arcs which join two points. Using Morse's hypotheses, we derive these relations rather simply from well known continuity properties of the solutions of differential equations, without making use of the critical point theory or $n$-dimensional analysis situs. It should be stated, however, that Morse has since proved the relations without

* Presented to the Society, September 7, 1928.

$\dagger \mathrm{M}$. Morse, The foundations of a theory in the calculus of variations in the large, Transactions of this Society, vol. 30 (1928), pp. 213-274.

$\ddagger$ M. Morse, Relations between the critical points of a real function of $n$ independent variables, Transactions of this Society, vol. 27 (1925), pp. 345396. 
the field hypothesis* and has also announced results for the $n$-dimensional calculus of variations problem. It seems improbable that our method can be generalized to treat these problems.

The method here presented is of interest, however, because in this case, it makes possible the statement of the relations in a somewhat more restrictive form than that given by Morse, in fact, in a form which immediately gives a new existence theorem. Moreover, our method gives an insight into the relative positions of the extremal arcs of different type, and is thus supplementary to the critical point method.

2. Hypotheses and Relations of Morse. Let the given integral be

$$
\int_{t_{0}}^{t_{1}} F(x, y, \dot{x}, \dot{y}) d t .
$$

I. We assume that the function $F(x, y, \dot{x}, \dot{y})$ is positively homogeneous of the first degree in $\dot{x}$ and $\dot{y}$, and analytic in all its arguments for $(x, y)$ any point interior to an open region $S$ of the $(x, y)$ plane and $\dot{x}$ and $\dot{y}$ any two numbers not both zero. We also assume that for the same arguments, $F_{1}(x, y, \dot{x}, \dot{y})>0$.

II. Let there be given a closed region $S_{1}$, interior to $S$, bounded by a simple closed curve $\beta$ consisting of a finite number of analytic arcs. We assume that $\beta$ is extremal-convex in the following sense: (1) The interior angles at the vertices of $\beta$ all lie between 0 and $\pi ;(2)$ An extremal tangent to any arc of $\beta$ lies outside of $S_{1}$ in the neighborhood of the point of contact.

III. We assume that $S_{1}$ is covered in a one-to-one manner by a proper field of extremals of the form

$$
x=h(u, v), \quad y=k(u, v),
$$

where $u$ is the parameter, and $v$ is the arc length measured along the extremals, and where at every point $(u, v)$ that corresponds to a point $(x, y)$ in $S_{1}$, the functions (2) are single-valued and analytic in $u$ and $v$, and

\footnotetext{
* See Morse, first paper, loc. cit., footnote, p. 223.
} 


$$
D=\left|\begin{array}{ll}
h_{u} & h_{v} \\
k_{u} & k_{v}
\end{array}\right| \neq 0 .
$$

IV. We assume that the problem is reversible.*

The region $S_{1}$ in the $(x, y)$ plane corresponds to a closed, extremal-convex region $R$ in the $(u, v)$ plane, whose boundary $\gamma$ is a simple closed curve consisting of a finite number of analytic arcs. If $\ddot{u}>0$ and we let $t=u$, the integral (1) can be put into non-parametric form with an integrand, $f\left(u, v, v^{\prime}\right)$, where $v^{\prime}=d v / d u$. The non-parametric problem is regular $\left(f_{v^{\prime} v^{\prime}}>0\right)$. The field extremals become the lines $u=$ constant, which of course are not included among the solutions of the non-parametric problem. All other extremals of the parametric form are included in the non-parametric problem because of the assumption of reversibility.

Morse proves a lemma (p. 224) which we take over. It amounts to the statement that in the simply-connected region $R$, no line $u=$ constant cuts the boundary $\gamma$ in more than two points.

In $R$, to the extremals $u=$ constant, no other extremals can be tangent. Hence, any extremal arc, other than $u=$ constant, is representable in the form $v=M(u)$, where $M(u)$ is analytic for that part of the arc which lies within $R$.

Let $A$ and $B$ be any two points, within or on the boundary of $R$, and not on the same line $u=$ constant. We state two preliminary results: (a) There exists at least one extremal arc which joins $A$ to $B$ in $R$ and which has no points on the boundary of $R$, with the possible exception of $A$ and $B$; (b) The points $A$ and $B$ can be joined by at most a finite number of extremal arcs in $R$. We shall prove (a) in $\$ 3$, while (b) is proved in Morse's paper (p. 226) without the use of the critical point theory.

We consider the set $G$ of $\operatorname{arcs}$ which join $A$ to $B$ in $R$. For the present, we assume that there exists no arc in $G$ upon which $B$ is conjugate to $A$. Each arc in $G$ has a type number

\footnotetext{
* See Morse, loc. cit., p. 223.
} 
$k$ which will be defined to be equal to the number of points on the arc conjugate to $A$. Let $m$ be the maximum of the type numbers $k$ and $M_{k}(k=1,2, \cdots, m)$ be the number of extremal arcs in $G$ of type $k$. The relations of Morse are then the following:

$$
\left\{\begin{array}{l}
M_{0} \geqq 1 \\
M_{0}-M_{1} \leqq 1 \\
\cdot \cdot \cdot \cdot \\
(-1)^{m-1}\left[M_{0}-M_{1}+\cdots+(-1)^{m-1} M_{m-1} \geqq 1,\right. \\
M_{0}-M_{1}+\cdots+(-1)^{m} M_{m}=1 .
\end{array}\right.
$$

3. A Preliminary Theorem. Morse gives the following consequence of the assumptions of $\$ 2$. There exists a positive constant e so small that any two points in $R$ whose abscissae differ in absolute value by less than e, can be joined by a unique extremal arc, all of whose points (except possibly the end points) lie within $R$.

We take over this result and show that it follows that, if $A$ and $B$ are any two points in $R$, not on the same line $u=$ constant, there exists at least one extremal arc $E$ which joins $A$ to $B$ in $R$, and that no points of $E$, except possibly $A$ and $B$, lie on the boundary $\gamma$ of $R$. Let $A$ and $B$ be represented by $\left(u_{0}, v_{0}\right)$ and $\left(u_{1}, v_{1}\right)$, respectively, $u_{0}<u_{1}$. By virtue of the result just stated, a finite number $p$ of lines of the form $u=$ constant can be chosen between $u=u_{0}$ and $u=u_{1}$ such that, if $p$ arbitrary points are chosen in $R$, one on each of these lines, there is determined a unique chain of extremal arcs, which joins $A$ to $B$. The value of the fundamental integral taken along such chains becomes a single-valued continuous function of the $p$ ordinates. The domain of definition is closed in each of the variables. Hence there exists a set of ordinates for which the integral takes on its minimum value. The extremal arcs of the corresponding chain join on to each other so as to form a single extremal arc $E$ from $A$ to $B$, since in a regular problem a curve with corners cannot minimize the integral. Now $E$ has no points 
on the boundary $\gamma$ with the possible exception of $A$ and $B$. This statement follows from the extremal-convex boundary and from the impossibility of corners.

We now prove the following theorem.

Theorem 1. If there exists in $R$, joining $A$ to $B$, an extremal arc $E_{0}$ of type $n>0$, there exist in $R$, joining $A$ to $B$, at least two other extremal arcs, one of which lies above $E_{0}$, the other below $E_{0}$.

Proof. Let $E_{0}$ be extended in both senses until it intersects $\gamma$, the boundary of $R$, thus dividing $R$ into two regions, $R_{1}$ and $R_{2}$. Now $R_{1}$ is extremal-convex in a somewhat broader sense than that of $\$ 2 \mathrm{II}$, in that part of the boundary is itself an extremal arc. But as before, $A$ and $B$ can be joined in $R_{1}$ by an extremal arc $E$ which minimizes the integral in comparison with all other arcs which join $A$ to $B$ in $R_{1}$. The points of $E$ lie within $R_{1}$ or on its boundary. But $E$ cannot coincide with $E_{0}$ because the latter contains at least one point conjugate to $A$ by hypothesis and hence cannot minimize the integral. In fact, it is readily proved that $E$ has no points except $A$ and $B$ in common with the boundary of $R_{1}$. A similar argument applies to $R_{2}$.

4. The Extremals through $A$. Let $A$ and $B$ be represented by $\left(u_{0}, v_{0}\right)$ and $\left(u_{1}, v_{1}\right)$, respectively. Let $E_{0}$ be an extremal arc in $G$ of type $n \geqq 0$. We consider the family of extremals through $A$. Any such extremal $E$ is defined by the angle $\alpha$, measured from $E_{0}$ to $E$ at $A$, since in a regular problem there exists* one and only one extremal through a given point with a given slope. Hence the family may be represented by

$$
v=v(u, \alpha) .
$$

Suppose that a particular extremal $E$ of (4) makes an angle of $\alpha_{0} \neq 0$ with $E_{0}$ at $A$, and intersects $E_{0}$ exactly $r$ times between $A$ and $B$. The abscissae of these points of

* Bolza, Vorlesungen uber Variationsrechnung, 1909, pp. 184-185. 
intersection will be denoted by $U_{i}(i=1,2, \cdots, r)$, where for each $i, u_{0}<U_{i}<u_{1}$. The number of intersections $r$ will depend upon $\alpha_{0}$. An arc $A P_{i}$ of $E$ has no points on the boundary of $R$, except possibly $A$. This follows from the regularity of the problem (extremals have no corners) and from the extremal-convex nature of the boundary. For $\alpha$ sufficiently near $\alpha_{0}$ and $u_{0}<u \leqq U_{i}$, the extremals (4) lie entirely within $R$, since $v(u, \alpha)$ is a continuous function* of $\alpha$. Let $E_{0}$ be represented by $v=f(u), u_{0} \leqq u \leqq u_{1}$. Then at $P_{i}$ we have

$$
v\left(U_{i}, \alpha_{0}\right)=f\left(U_{i}\right), \quad(i=1,2, \cdots, r) .
$$

Since* $v(u, \alpha)$ and $f(u)$ are of class $C^{\prime}$ and $v_{u}\left(U_{i}, \alpha_{0}\right) \neq f^{\prime}\left(U_{i}\right)$ for $\alpha_{0} \neq 0$, the implicit function theorem is applicable and for $\alpha$ near $\alpha_{0}$ the abscissa $U_{i}$ of the $i$ th point of intersection of the extremals (4) with $E_{0}$ is a continuous differentiable function of $\alpha$. In fact, for $\alpha$ near $\alpha_{0}$, we have identically

$$
v\left[U_{i}(\alpha), \alpha\right]=f\left[U_{i}(\alpha)\right],(i=1,2, \cdots, r) .
$$

Differentiating with respect to $\alpha$, we have

$$
v_{u}\left[U_{i}(\alpha), \alpha\right] \cdot U_{i}^{\prime}(\alpha)+v_{\alpha}\left[U_{i}(\alpha), \alpha\right]=f^{\prime}\left[U_{i}(\alpha)\right] \cdot U_{i}^{\prime}(\alpha) .
$$

Rearranging and omitting arguments, we obtain

$$
v_{\dot{\alpha}}=U_{i}^{\prime}\left(f^{\prime}-v_{u}\right) \text {. }
$$

Here $f^{\prime}$ and $v_{u}$ represent the slopes of $E_{0}$ and $E$ respectively, at their $i$ th point of intersection.

By definition, any function $U_{i}(\alpha)$ will be restricted by the inequality $u_{0}<U_{i}(\alpha) \leqq u_{1}$.

For $\alpha$ sufficiently small, exactly $n$ functions $U_{i}(\alpha)$ exist (that is, $r=n$ ) and the abscissae of the conjugate points on $E_{0}$ are given $\dagger$ by $U_{i}(0),(i=1,2, \cdots, n)$. For numerically larger values of $\alpha$, some of these functions may cease to be defined, or on the other hand, functions $U_{i}(\alpha)$ may exist for $i>n$.

If $U_{k}\left(\alpha_{0}\right)<u_{1}$, we have seen that the function $U_{k}(\alpha)$ is defined for $\alpha$ sufficiently near $\alpha_{0}$. The ends of the intervals

* Bolza, loc. cit., p. 73.

† Bolza, loc, cit., p. 79. 
of definition of $U_{k}(\alpha)$ must therefore correspond to values of $\alpha$ for which $U_{k}(\alpha)=u_{1}$.

Since the problem is regular, no extremal is tangent to any other in $R$. The points of intersection of two extremals are therefore discrete. Hence if $U_{k}(\alpha)$ is defined on a certain $\alpha$ interval, the functions $U_{i}(\alpha), i<k$, are also defined on this interval and

$$
U_{i}(\alpha)<U_{j}(\alpha), i<j \leqq k .
$$

The necessary and sufficient condition that an extremal $E$, through $A$, pass through $B$ is that

$$
U_{k}(\alpha)=u_{1},
$$

for some integer $k$. For values of $\alpha$ for which (6) holds, $U_{k}(\alpha)$ will have in general only a one-sided derivative.

In discussing equation (5), with $i$ replaced by $k$, it will suffice for our purposes to consider the case $\alpha>0$. Then, if $k$ is odd, $v_{u}<f^{\prime}$ and $v_{\alpha}$ takes the sign of $U_{k}^{\prime}$. If $k$ is even, $v_{u}>f^{\prime}$ so that $v_{\alpha}$ and $U_{k}^{\prime}$ have opposite signs.

Now $v_{\alpha}(0, \alpha)=0$ and $v_{\alpha u}(0, \alpha)>0$. Hence the conjugate points to $A$ on $E$ are given* by those values of $u$ for which $v_{\alpha}(u, \alpha)=0$. Then if $v_{\alpha}>0$ for $u=u_{1}$, there exist an even number of points conjugate to $A$ between $A$ and $B$; if $v_{\alpha}<0$, an odd number. From (5) therefore, we obtain the following theorem.

THEOREM 2. Let the functions $U_{i}(\alpha)$ be defined relative to an extremal arc $E_{0}$, which joins $A$ to $B$. Suppose $E$ is an extremal arc which joins $A$ to $B$ and which makes an angle $\alpha_{0}>0$ with $E_{0}$ at $A$. Let $n$ be the type of $E$ and $k$ the integer such that $U_{k}\left(\alpha_{0}\right)=u_{1}$, the abscissa of $B$. Then $n$ is odd if $U_{k}^{\prime}\left(\alpha_{0}\right)>0$ and $k$ is even, or if $U_{k}^{\prime}\left(\alpha_{0}\right)<0$ and $k$ is odd; $n$ is even if $U_{k}^{\prime}\left(\alpha_{0}\right)>0$ and $k$ is odd, or if $U_{k}^{\prime}\left(\alpha_{0}\right)<0$ and $k$ is even. Finally, $B$ is conjugate to $A$ if $U_{k}^{\prime}\left(\alpha_{0}\right)=0$, for any $k$.

5. Types of Adjacent Arcs. Now let the extremal arcs of $G$ be arranged in order of their slopes at $A$. Two arcs

* Bolza, loc. cit., p. 78. 
which are consecutive in this order will be said to be adjacent to each other. We now prove the following theorem.

Theorem 3. Adjacent extremal arcs in $G$ differ in type by unity.

Let $E$ and $\bar{E}$ be the adjacent extremal arcs, $E$ being that arc which has the lesser slope at $A$. Let $n$ and $\bar{n}$ be the type of $E$ and $\bar{E}$ respectively. Suppose first that the functions $U_{i}(\alpha)$ are defined relative to $E$.

Since $E$ is of type $n$, we know that $U_{n}(0)<u_{1}$ and that $U_{n+1}(\alpha)$ is not defined for $\alpha$ sufficiently small. Let $\alpha_{0}$ be the angle at $A$ from $E$ to $\bar{E}$. Then $U_{k}\left(\alpha_{0}\right)=u_{1}$ for some integer $k$, which it remains to determine. Now $U_{n}\left(\alpha_{0}\right)$ is defined. Otherwise we would have $U_{n}(\alpha)=u_{1}$ for some positve $\alpha<\alpha_{0}$, which would imply that $\bar{E}$ was not adjacent to $E$. There are therefore two cases:

$$
\begin{aligned}
& U_{n}\left(\alpha_{0}\right)=u_{1}, \\
& U_{n}\left(\alpha_{0}\right)<u_{1},
\end{aligned}
$$

CASE A. $U_{n}\left(\alpha_{0}\right)=u_{1}$. We have assumed that $B$ is not conjugate to $A$ on $\bar{E}$. Then by Theorem $2, U_{n}^{\prime}\left(\alpha_{0}\right) \neq 0$. Since $U_{n}(\alpha)<u_{1}$ for $0 \leqq \alpha<\alpha_{0}$, it now follows that $U_{n}^{\prime}\left(\alpha_{0}\right)>0$.

CASE B. $U_{n}\left(\alpha_{0}\right)<u_{1}$. Here $U_{n+1}\left(\alpha_{0}\right)$ must exist. If $U_{n+1}\left(\alpha_{0}\right)<u_{1}$, there exists a positive $\alpha<\alpha_{0}$ for which $U_{n+1}(\alpha)=u_{1}$, which implies that $\bar{E}$ and $E$ are not adjacent. Hence $U_{n+1}\left(\alpha_{0}\right)=u_{1}$. Since $U_{n+1}^{\prime}\left(\alpha_{0}\right) \neq 0$ and $U_{n+1}(\alpha)$ is not defined for $0 \leqq \alpha<\alpha_{0}$, it follows that $U_{n+1}^{\prime}\left(\alpha_{0}\right)<0$. Thus we must have one of the following possibilities:

$$
\begin{aligned}
U_{n}\left(\alpha_{0}\right) & =u_{1}, \quad U_{n}^{\prime}\left(\alpha_{0}\right)>0 ; \\
U_{n+1}\left(\alpha_{0}\right) & =u_{1}, \quad U_{n+1}^{\prime}\left(\alpha_{0}\right)<0 .
\end{aligned}
$$

Using Theorem 2, with $n=\bar{n}$ and $k=n$ and $n+1$ respectively, we now see that, for either (A) or (B), if $n$ is odd, $\bar{n}$ is even; while if $n$ is even, $\bar{n}$ is odd. Hence in particular, $n \neq \bar{n}$.

We now reverse the roles of $E$ and $\bar{E}$. Let the functions 
$\bar{U}_{i}(\alpha)$ be defined relative to $\bar{E}$ in the same manner that the functions $U_{i}(\alpha)$ were defined relative to $E$.

For Case A, we have

$$
\bar{U}_{n}\left(-\alpha_{0}\right)=U_{n}\left(\alpha_{0}\right)=u_{1},
$$

which implies that

$$
\bar{U}_{i}\left(-\alpha_{0}\right)<u_{1}, \quad(i<n) .
$$

Since the functions $\bar{U}_{i}(\alpha)$ are continuous and bounded, it follows that

$$
\bar{U}_{i}(0)<u_{1}, \quad(i<n) .
$$

Perhaps also $\bar{U}_{n}(0)<u_{1}$, but certainly $\bar{U}_{n+1}(0)$ is not defined since $E$ is adjacent to $\bar{E}$. Since the numbers $\bar{U}_{i}(0)$ are the abscissae of the conjugate points to $A$ on $\bar{E}$, the type $\bar{n}$ of $\bar{E}$ is either $n$ or $n-1$. But we proved that $\bar{n} \neq n$, so that for Case $\mathrm{A}$ there remains the single possibility $\bar{n}=n-1$.

A similar argument shows that for Case $\mathrm{B}, \bar{n}=n+1$. Combining the results of both cases, $\bar{n}=n-1$ or $n+1$, as the theorem states.

6. Proof of Number Relations. Let the extremal arcs which join $A$ to $B$ in $R$, be arranged in order of their slopes at $A$. The first and last must be of type zero. Otherwise, according to Theorem 1, there would exist extremal arcs of $G$ whose initial slopes were respectively less than and greater than the initial slopes of these arcs.

Since adjacent arcs differ in type by unity, we see that in order that the first and last shall be of type zero, the total number of extremal arcs in $G$ must be odd. Let us remove from $G$ either the first or last arc and group the remaining ones into adjacent pairs. If $m$ is the maximum type number which occurs in $G$, the integers $M_{k}$ must be of the following form:

$$
\left\{\begin{array}{l}
M_{0}=1+p_{1}, \\
M_{1}=p_{1}+p_{2}, \\
\cdot \cdot \cdot \cdot p_{i} \geqq 1,(i=1,2, \cdots, m), \\
M_{m-1}=p_{m-1}+p_{m}, \\
M_{m}=p_{m},
\end{array}\right.
$$


where the integers $p_{i}$ represent the total number of pairs, which comprise extremal arcs of types $i$ and $i-1$. It is readily seen that there exists at least one pair for each value of $i$.

By addition and subtraction of the equations of (7), we obtain the relations (3) of Morse. But a stronger form of these relations follows if we substitute $p_{i}=q_{i}+1$ in (7). Then

$$
\left\{\begin{array}{l}
M_{0}=2+q_{1}, \\
M_{1}=2+q_{1}+q_{2}, \\
\cdot \cdot \cdot \cdot q_{i} \geqq 0,(i=1,2, \cdots, m), \\
M_{m-1}=2+q_{m-1}+q_{m}, \\
M_{m}=1+q_{m} .
\end{array}\right.
$$

Now by addition and subtraction, we obtain the following theorem.

THEOREM 4. If $M_{k}$ represents the number of extremal arcs of type $k$ which join $A$ to $B$, and if $m$ is the maximum of the types $k$, then

$$
\left\{\begin{array}{l}
M_{0} \geqq 2 \\
M_{0}-M_{1} \leqq 0 \\
\cdot \cdot \cdot \cdot \cdot \\
M_{0}-M_{1}+\cdots+(-1)^{m} M_{m}=1
\end{array}\right.
$$

where the next to the last relation is

$$
M_{0}-M_{1}+\cdots+(-1)^{m-1} M_{m-1} \geqq 2 \text { or } \leqq 0,
$$

according as $m$ is even or odd.

From $\left(7^{\prime}\right)$ we have the following new theorem.

THEOREM 5. If $M_{k}$ represents the number of extremal arcs of type $k$ which join $A$ to $B$ and if $m$ is the maximum of the types $k$, then

$$
\left\{\begin{array}{l}
M_{i} \geqq 2, \quad(i=1,2, \cdots, m-1), \\
M_{m} \geqq 1 .
\end{array}\right.
$$

We have as an immediate corollary, 
Corollary. If there exists in $G$ an extremal arc of type $m$, there exist in $G$ at least $2 m$ other extremal arcs.

It is of interest to consider the geodesics, on a torus generated by revolving a circle $C$ of radius $a$ about an axis in its plane which does not intersect it. Let $u$ measure the angle through which $C$ has been revolved from an initial position, and let $v$ represent arc length measured along $V$ from the point farthest from the axis. Let $A\left(u_{0}, 0\right)$ and $B\left(u_{1}, 0\right)$ be the end points of a segment of the geodesic $v=0$ of type $m$. The geodesics which join $A$ to $B$ and lie in the $u v$ plane within the rectangle bounded by $u=u_{0}, u=u_{1}, v=\pi a, v=-\pi a$ are then exactly $2 m+1$ in number and the integers $M_{i}$ satisfy the equalities in (9). By choosing the two points sufficiently far apart, $m$ may be made arbitrarily large.

7. A Restriction Removed. It is now necessary to remove the restriction so far imposed, namely, that on no arc in $G$ is $B$ conjugate to $A$. Here we adopt the procedure of Morse (pp. 231-232), merely stating the results. A point $B^{\prime}$ may be chosen near $B$ and not on an envelope of the extremals through $A$. For the extremal arcs which join $A$ to $B^{\prime}$, relations (8) hold. Now allow $B^{\prime}$ to approach $B$ continuously in such a manner as to avoid envelopes. It will be found that relations (8) continue to hold for $B=B^{\prime}$, provided that certain conventions are adopted as to the types of the extremal arcs in $G$. These conventions are as follows. Let $\mathrm{g}$ be an extremal arc joining $A$ to $B$ on which there are $m$ points conjugate to $A$ prior to $B$. If $B$ is not conjugate to $A, g$ is to be counted as one arc of type $k=m$. If $B$ is conjugate to $A$ and $B$ is an ordinary point on the $(m+1)$ st envelope $T$ of the extremals through $A, g$ is to be counted as two extremal arcs of types $k=m+1$ and $k=m$, respectively. If $B$ is conjugate to $A$ and $T$ has a cusp at $B$ which opens toward $A, g$ is to be counted as one extremal arc of type $k=m+1$. If $T$ has a cusp at $B$ which opens away from $A, g$ is to be counted as one arc of type $k=m$.

Williams College 